\title{
Cholinergic Regulation of the Suprachiasmatic Nucleus Circadian Rhythm via a Muscarinic Mechanism at Night
}

\author{
Chen Liu ${ }^{1}$ and Martha U. Gillette ${ }^{1,2,3}$ \\ ${ }^{1}$ Neuroscience Program, and Departments of ${ }^{2}$ Cell and Structural Biology and ${ }^{3 P h y s i o l o g y, ~ U n i v e r s i t y ~ o f ~ I l l i n o i s ~ a t ~}$ \\ Urbana-Champaign, Urbana, Illinois 61801
}

In mammals, the suprachiasmatic nucleus (SCN) is responsible for the generation of most circadian rhythms and for their entrainment to environmental cues. Carbachol, an agonist of acetylcholine (ACh), has been shown to shift the phase of circadian rhythms in rodents when injected intracerebroventricularly. However, the site and receptor type mediating this action have been unknown. In the present experiments, we used the hypothalamic brain-slice technique to study the regulation of the SCN circadian rhythm of neuronal firing rate by cholinergic agonists and to identify the receptor subtypes involved. We found that the phase of the oscillation in SCN neuronal activity was reset by a 5 min treatment with a carbachol microdrop ( $1 \mu \mathrm{l}, 100 \mu \mathrm{M})$, but only when applied during the subjective night, with the largest phase shift (+6 hr) elicited during the middle of the subjective night. This effect also was produced by $\mathrm{ACh}$ and two muscarinic receptor (mAChR) agonists, muscarine and $\mathrm{McN}-\mathrm{A}-343$ (M1-selective), but not by nicotine. Furthermore, the effect of carbachol was blocked by the $\mathrm{mAChR}$ antagonist atropine $(0.1 \mu \mathrm{M})$, not by two nicotinic antagonists, dihydro- $\beta$-erythroidine $(10 \mu \mathrm{M})$ and $\alpha$-tubocurarine $(10 \mu \mathrm{M})$. The M1-selective mAChR antagonist pirenzepine completely blocked the carbachol effect at $1 \mu \mathrm{M}$, whereas an M3-selective antagonist, 4,2-(4,4'-diacetoxydiphenylmethyl)pyridine, partially blocked the effect at the same concentration. Ihese results demonstrate that carbachol acts directly on the SCN to reset the phase of its firing rhythm during the subjective night via an $\mathrm{M1}$-like mAChR.

Key words: acetylcholine; carbachol; McN-A-343; circadian rhythm; suprachiasmatic nucleus; muscarinic receptor; pirenzepine; atropine; 4-DAMP; nicotine; dihydro- $\beta$-erythroidine; d-tubocurarine
The suprachiasmatic nucleus (SCN) of the hypothalamus has been identified as a primary pacemaker for circadian rhythms in mammals. Circadian rhythms are synchronized to environmental cues such as the solar light-dark cycle by afferent signals that have complex interactions with the SCN pacemaker. Brief light pulses administered to animals maintained in continuous darkness are capable of shifting the phase of their behavioral and physiological rhythms in a direction that depends on the time of administration of the pulse (De Coursey, 1964). Pharmacological studies have revealed a number of chemical agents that phase-shift circadian rhythms in rodents (for review, see Rusak and Bina, 1990). Carbachol, an agonist of acetylcholine ( $\mathrm{ACh}$ ), has been reported to induce phase shifts in the circadian rhythm of pineal $N$-acetyltransferase (NAT) activity in rats and locomotor activity in mice and hamsters. Phase shifts were similar to those induced in response to brief light pulses, namely, delays in early subjective night and advances in late subjective night (Zatz and Brownstein, 1979; Zatz and Herkenham, 1981; Earnest and Turek, 1983, 1985; Wee et al., 1992). However, Meijer et al. (1988) reported that carbachol induced only phase advances. Because almost all of these in vivo studies used intracerebroventricular injection, the site of action could not be determined.

\footnotetext{
Received June 27, 1995; revised Oct. 13, 1995; accepted Oct. 17, 1995.

This work was supported by National Institutes of Health Grants NS22155 and NS33240, a University of Illinois Campus Research Board Award, and a Beckman Scholar Award to M.U.G. We thank Dr. Eve A. Gallman for reading this manuscript.

Correspondence should be addressed to Martha U. Gillette, Department of Cell and Structural Biology, University of Illinois, 506 Morrill Hall, 505 South Goodwin Avenue, Urbana, IL 61801.

Dr. Liu's present address: Laboratory of Developmental Chronobiology, Children's Service, GRJ 1226, Massachusetts General Hospital, Boston, MA 02114.

Copyright $(C) 1996$ Society for Neuroscience $0270-6474 / 96 / 160744-08 \$ 05.00 / 0$
}

The possibility of a direct regulation of the SCN by cholinergics is suggested by neuroanatomical studies. Both the $\mathrm{ACh}$ degrading enzyme acetylcholinesterase and the biosynthetic enzyme choline acetyltransferase are present in the SCN and its surrounding neuropil (Brownstein et al., 1975; van den Pol and Tsujimoto, 1985; Ichikawa and Hirata, 1986; Rao et al., 1987; Tago et al., 1987). Using monoclonal antibodies against the muscarinic and nicotinic $\mathrm{ACh}$ receptors (mAChR, $\mathrm{nAChR}$ ), both antigens were identified within the rat $\mathrm{SCN}$ (van der Zee et al., 1991). More recently, the sites of cholinergic neurons that project to the SCN area have been identified (Bina et al., 1993) and include the basal nuclear complex of the forebrain and the laterodorsal tegmental, pedunculopontine tegmental, and parabigeminal nuclei of the brainstem. Although the functional significance of these pathways remains to be determined, it has been speculated that they are part of a feedback loop between the regulation of sleep/arousal and the circadian timing system (Bina et al., 1993).

The receptor mechanisms underlying the effect of carbachol on circadian rhythms have been uncertain (for review, see Rusak and Bina, 1990). Although nicotinic sites appeared to mediate the light-like influence of carbachol on pineal NAT activity, a role for muscarinic receptors was not ruled out (Zatz and Brownstein, 1981). In a recent study using the brain-slice preparation, bathapplied nicotine, but not carbachol, was reported to phaseadvance the SCN neuronal activity rhythm (Trachsel et al., 1995). A majority of SCN neurons were excited by iontophoresis of $\mathrm{ACh}$ (Nishino and Koizumi, 1977) or by systemic injection of cholinergic agonists (Miller et al., 1987) in rats. However, the effects of $\mathrm{ACh}$ on the activity of the SCN neurons recorded in vitro were predominantly inhibitory (Shibata et al., 1983; Kow and Pfaff, 
1984) and could be blocked by atropine (Kow and Pfaff, 1984), indicating the involvement of mAChRs.

Spontaneous neuronal firing of the SCN in the hypothalamic brain slice is patterned, appearing as a circadian oscillation (Green and Gillette, 1982) that is stable for at least three cycles (Prosser and Gillette, 1989). In the present study, we evaluated the temporal sensitivity of this rhythm of neuronal firing in the $\mathrm{SCN}$ to direct cholinergic stimulation in vitro. We sought to answer the following questions. (1) Can cholinergic agonists act directly on the SCN to alter the phase of the neuronal activity rhythm in the isolated rat SCN? (2) If so, which receptors are involved in the cholinergic regulation?

Preliminary findings of this study have been reported in abstract form (Liu and Gillette, 1994).

\section{MATERIALS AND METHODS}

Animals and brain-slice preparation. Eight-week-old male and female Long-Evans rats from our inbred colony were used in this study. Animals were maintained and tissue-prepared in full accord with institutional and federal guidelines for the hunane treatment of animals. The animals were kept on a schedule of $12 \mathrm{hr}$ of light and $12 \mathrm{hr}$ of darkness and fed ad libitum. The animals were killed during the "lights-on" period of the $24 \mathrm{hr}$ cycle. This was necessary to avoid phase-shifting effects, which have been shown to occur with manipulations at night (Gillette, 1986). The animals were decapitated, and the brain was dissected quickly from the skull. The brain then was sectioned manually to form a block of tissue containing the hypothalamic region. This block of tissue was transferred to a mechanical tissue chopper on which $500 \mu \mathrm{m}$ coronal slices were made.

The hypothalamic slices containing the SCN then were transferred to the brain-slice dish, in which they were maintained for up to $3 \mathrm{~d}$. The brain-slice dish was modeled after Hatton et al. (1980). The dish consisted of an inner chamber and an outer chamber. The outer chamber was filled with d- $\mathrm{H}_{2} \mathrm{O}$ warmed to $37^{\circ} \mathrm{C}$ and bubbled continuously with $95 \%$ $\mathrm{O}_{2} / 5 \% \mathrm{CO}_{2}$. The slice in the inner chamber was perifused at $35 \mathrm{ml} / \mathrm{hr}$ with warmed, oxygenated Earle's balanced salt solution [(in $\mathrm{mM}$ ) $1.8 \mathrm{CaCl}_{2}, 5.3$ $\mathrm{KCl}, 0.8 \mathrm{MgSO}_{4}, 117 \mathrm{NaCl}$ and $1 \mathrm{NaH}_{2} \mathrm{PO}_{4} \cdot \mathrm{H}_{2} \mathrm{O}$; Gibco, Gaithersburg, MD] supplemented with $24.6 \mathrm{~mm}$ glucose, $26.3 \mathrm{~mm}$ sodium bicarbonate, and $0.005 \%$ gentamycin, $\mathrm{pH} 7.3-7.4$.

Extracellular electrical recording and data analysis. Under these constant conditions of incubation in a brain slice, the SCN generates a near $24 \mathrm{hr}$ oscillation in firing activity of the neuronal ensemble (Green and Gillette, 1982). The unperturbed sinusoidal pattern of ensemble activity is predictably high during the subjective day, peaking mid-day near circadian time 7 (CT 7, or $7 \mathrm{hr}$ after "lights on" in the $24 \mathrm{hr}$ lighting schedule in the rat colony), and low during subjective night (Gillette and Prosser, 1988), so that measurement of the time-of-peak can be used as an accurate assessment of the phase of the oscillation (Gillette et al., 1995).

The details of this sampling method and validation of this technique for determining the phase of the SCN circadian rhythm of ensemble neuronal firing rate have been published previously (Prosser and Gillette, 1989). Briefly, extracellular recordings from single cells were made using glass microelectrodes filled with $5 \mathrm{M} \mathrm{NaCl}$. An electrode was lowered into the SCN using a hydraulic microdrive until the signal from a single cell was encountered. Electrical signals exceeding twice the level of the background noise were isolated using a window discriminator, observed for stability over at least $2 \mathrm{~min}$, and sent to a computer for counting. The firing rate of each cell was monitored over two consecutive 2 min periods, and the values were averaged to determine the mean firing frequency of that cell at that circadian time. The electrode then was repositioned arbitrarily within the SCN until another cell was encountered. An allempt was made to sample all areas of the nucleus. In each experiment, 40-70 cells were recorded over a period of $8-12 \mathrm{hr}$.

The firing rates of individual $\mathrm{SCN}$ neurons recorded during each experiment were grouped into $2 \mathrm{hr}$ bins based on the circadian time at which they were counted. Successive bins were offset by $15 \mathrm{~min}$ to produce a running average. This procedure smooths the measurements made on individual neurons, which show a range of activities, and permits better resolution of the circadian time-of-peak in the activity rhythm of the neuronal ensemble. The time-of-peak then was determined by visual inspection of a graph of these values for the symmetrically highest point.
To simplify the illustrations, only the $2 \mathrm{hr}$ means offset by $1 \mathrm{hr}$ are presented in Figures 1, 3, 5, and 6. The phase shift was determined by comparing the time-of-peak electrical activity in drug-treated slices with that of media- and vehicle-treated control slices. The effect on the time-of-peak of a single treatment on a single $\mathrm{SCN}$, the individual neuronal activities of which were monitored for $8-12 \mathrm{hr}$, constituted a single $n$ for each experiment presented here. Differences between groups were evaluated using Student's unpaired $t$ test. Because we did not observe any difference between male and female rats, the data from male and female rats were combined.

Experimental treatments. Slices were allowed to equilibrate for at least $2 \mathrm{hr}$ in the recording chamber before any chemical treatment. Cholinergic agonists and antagonists were dissolved in normal incubation medium. Before application, the test solution was adjusted to $\mathrm{pH}$ 7.4 and was warmed to $37^{\circ} \mathrm{C}$ and bubbled with $95 \% \mathrm{O}_{2} / 5 \% \mathrm{CO}_{2}$. At the circadian time selected for evaluation, perifusion was stopped, a $1 \mu \mathrm{l}$ drop of medium containing carbachol, $\mathrm{ACh}$, or other cholinergic agonist was applied to each SCN for $5 \mathrm{~min}$ and then washed away toward the optic chiasm, after which slice perifusion with normal medium was resumed. In the experiment using the antagonists, the normal bathing medium was replaced in $<2$ min with warmed, oxygenated medium, $\mathrm{pH}$ 7.4 , containing the inhibitory substance. After $10 \mathrm{~min}$, a drop of control medium or medium containing carbachol was applied as stated above. Five minutes later, the surface of the slice was rinsed with medium containing the antagonist and incubated for an additional $15 \mathrm{~min}$. The bath treatment medium then was exchanged completely for normal medium and perifusion was resumed. Electrical activity then was monitored during the subsequent 1 or 2 circadian cycles to determine the time of the peak in neuronal firing (see data analysis). Carbachol was from Sigma (St. Louis, MO); other agonists and antagonists were from Research Biochemicals (Natick, MA).

\section{RESULTS}

\section{Carbachol applied to the SCN resets the neuronal firing rhythm during subjective night}

The sensitivity of the isolated pacemaker to cholinergic resetting of its phase was examined by applying microdrops $(100 \mu \mathrm{M}, 1 \mu \mathrm{l})$ of the cholinergic agonist carbachol directly to each of the paired $\mathrm{SCN}$ in a hypothalamic brain slice for $5 \mathrm{~min}$. The rhythm of firing rate was examined the next day. When a microdrop containing 100 $\mu \mathrm{M}$ carbachol in medium was applied in the middle of the subjective day at CT 6 on day 1 , the subsequent peak in electrical activity occurred at CT $6.3 \pm 0.1(n=3)$ on day 2 . An example of the rhythm of one SCN treated at CT 6 appears in Figure 1A. This time-of-peak is not significantly different from media-treated controls (CT $6.8 \pm 0.1, p>0.05 ; n=5$ ). In contrast, when SCN were exposed to an identical treatment during mid-subjective night at CT 18 on day 1, the pattern of firing rate in the next cycle (day 2) was altered. Peak activity no longer occurred near CT 7 , the normal peak time, or the time in media-treated control; rather, the peak occurred early (Fig. $1 B)$, at CT $0.7 \pm 0.2(n=3)$ the next day. Compared with the time-of-peak on day 2 of media-treated control experiments (CT $6.8 \pm 0.1 ; n=5$ ), this represents a mean advance of the phase $(\phi A)$ of $6.1 \mathrm{hr}(p<0.0001)$. To determine whether these phase advances were stable, we monitored the electrical activity rhythm for a second cycle after treatment (day 3 in vitro). As shown in Figure $1 C$, the time of peak firing rate was again at CT 1.0 in the second cycle after treatment of carbachol at CT 18.

It is possible that carbachol diffused away from the site of application and the effect measured was attributable to its action on a structure in the hypothalamic slice outside the SCN. Therefore, we used a reduced slice preparation that contained only $\mathrm{SCN}$, the underlying optic chiasm, and a small rim of hypothalamic tissue. The time of peak activity recorded from reduced slices was unchanged from that of the normal slice preparation (Gillette and Reppert, 1987). When applied as a microdrop to 


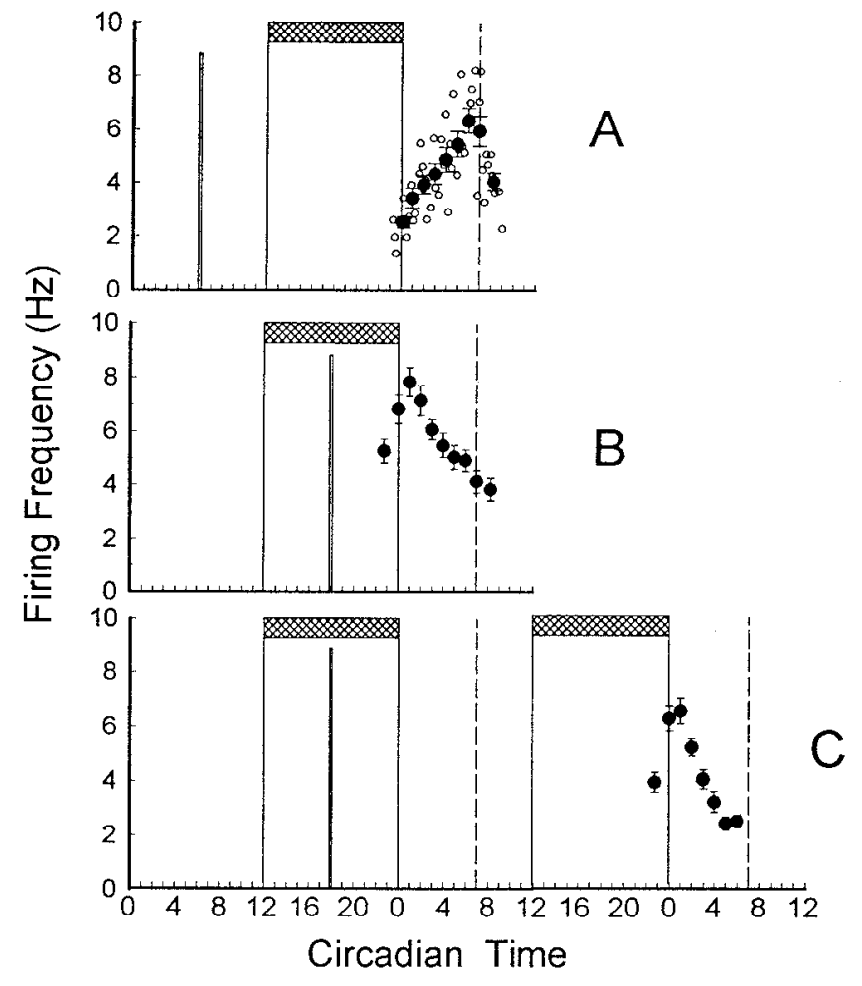

Figure 1. Phase-dependent effects of carbachol on the circadian rhythm of electrical activity. $A$, Carbachol treatment $(100 \mu \mathrm{M}$ in a $1 \mu 1$ droplet) at CT 6 on day 1 in vitro had little effect on the time-of-peak in neuronal activity on day 2 (peak at CT 6.25). $B$, The same carbachol treatment at CT 18 on day 1 shifted the electrical activity rhythm so that the time-of-peak activity on the second day in vitro occurred at CT 1.2 , which is $5.6 \mathrm{hr}$ earlier than normal. $C$, Electrical activity recorded in a separate slice treated at CT 18 on day 1 peaked at CT 1.0 on the third day. The time of the shifted peak in activity was consistent across experiments $(\phi A=+6.3 \pm 0.2 \mathrm{hr} ; n=3)$. The $2 \mathrm{hr}$ means $\pm \mathrm{SEM}$ of all single units recorded (solid circles) are plotted for each $2 \mathrm{hr}$ interval offset by $1 \mathrm{hr}$ to produce a running average that better discriminates the time-of-peak activity. Small open circles in $A$ (omitted in $B$ and $C$ ) represent the average firing rate of each neuron sampled. This permits visualization of the data on which $2 \mathrm{hr}$ means are based. Horizontal bars indicate subjective night (CT 12-24), the time of lights-off in the donor colony. Vertical open bars indicate the time of treatment. Dashed lines indicate the mean time-of-peak activity in control slices (CT $6.8 \pm 0.1$; $n=5$ ).

these SCN, carbachol still shifted the rhythm in the same way (peak $=\mathrm{CT} 0.8, \phi A=6 \mathrm{hr} ; n=2$ ). Thus, we conclude that carbachol stably phase-shifts the neuronal activity rhythm of the SCN via direct action on the SCN.

To characterize the differential temporal sensitivity of the pacemaker to carbachol more fully, we examined the effects of carbachol microdrops $(100 \mu \mathrm{M})$ applied for $5 \mathrm{~min}$ at various points in the circadian cycle. Because the advanced peak in firing activity appeared 6-7 hr after carbachol treatment at CT 18 , and activity peaked again 24 hr later, the pliase changes induced by carbachol appeared to be completed within $6-7 \mathrm{hr}$ after the treatment of carbachol. Therefore, the phase changes in this series of experiments were determined during the cycle after treatment. The resulting relationship between the circadian phase of treatment and the response of the pacemaker, a phase-response curve, is shown in Figure 2. When applied at points throughout the subjective day, carbachol induced little or no change $(+0.9 \pm 0.2 \mathrm{hr}$, between CT 0 and CT $12 ; n=7)$.

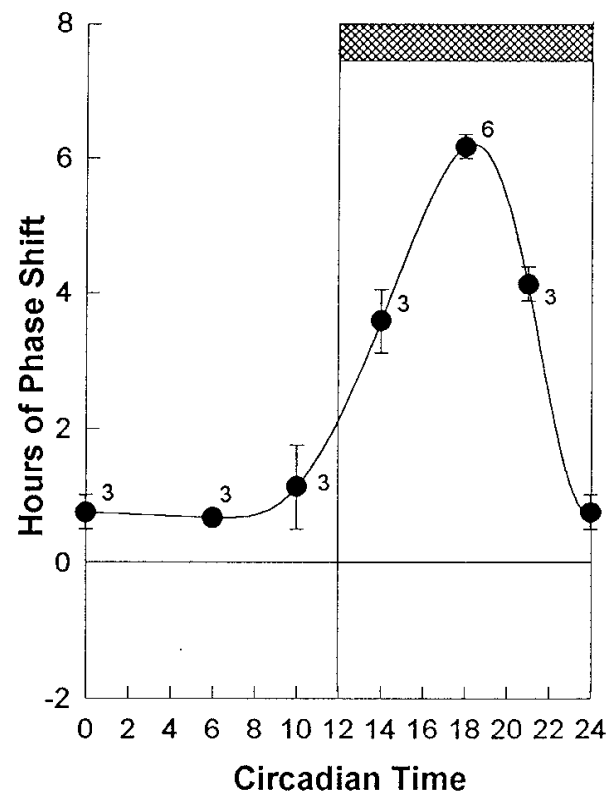

Figure 2. Relationship between the circadian time of carbachol treatment and its effect on the phase of the firing activity rhythm. The phase shifts induced by a 5 min exposure to $100 \mu \mathrm{M}$ carbachol in $1 \mu \mathrm{l}$ droplets at various circadian times were measured, and the average magnitudes of phase shifts \pm SEM $(n=3-6)$ were plotted. This figure is based on measurements across the $24 \mathrm{hr}$ circadian cycle with a total of 21 experiments. Experiments were performed at 12 circadian times, which were grouped in $2 \mathrm{hr}$ bins, and the means were plotted at the midpoint of the bin. The number of experiments in each bin is noted. For each experiment, the time-of-peak activity rhythm is based on the rhythm derived from measurements of 41-66 cells. Treatment during subjective night (horizontal bar) caused a significant phase advance in the peak of the circadian rhythm of electrical activity. The largest phase advance occurred at CT $17-19(+6.1 \pm 0.3 \mathrm{hr} ; n=6)$, whereas treatment during subjective daytime (CT $0-12$ ) had little effect on the rhythm.

However, the SCN circadian rhythms were reset significantly by carbachol throughout subjective night: the largest phase changes $(+6.1 \pm 0.3 \mathrm{hr} ; n=6)$ occurred when carbachol was applied between CT 17 and CT 19.

\section{The effect of carbachol is dose-dependent and mimicked by ACh and by muscarinic agonists, but not by nicotine}

Dose-response relationships were determined at CT 18, the time of maximal phase shift to carbachol. Examination of the doseresponse relationship for carbachol with the $5 \mathrm{~min}$ microdrop treatment shows that the half-maximal response occurred near 1 $\mu \mathrm{M}$, whereas the maximal response $(+6.3 \mathrm{hr})$ occurred at $10 \mu \mathrm{M}$. The neurotransmitter $\mathrm{ACh}$ induced a similar phase advance $(+5.3$ hr) at $0.1 \mu \mathrm{M}$. Effects of two muscarinic agonists, muscarine and the M1-selective $\mathrm{McN}-\mathrm{A}-343$, also were tested at a range of concentrations. They both induced a phase advance with the largest phase advances $(+6.5 \mathrm{hr}$ for McN-A-343 at $1 \mu \mathrm{M}$ and +6.3 hr for muscarine at $100 \mu \mathrm{M}$ ) within the amplitude range of those induced by carbachol (Figs. 3, 4). The $\mathrm{nAChR}$ agonist nicotine had no effect at concentrations of 1 and $10 \mu \mathrm{M}(n=3)$. At $100 \mu \mathrm{M}$ nicotine induced very small phase advances $(+1.2 \pm 0.3 \mathrm{hr} ; n=$ $3)$, which were statistically significant $(0.001<p<0.05$; Figs. 3 , 4). The relative potency of these agonists in shifting the SCN rhythm is as follows: $\mathrm{ACh}>\mathrm{McN}-\mathrm{A}-343>$ carbachol $\approx$ musca- 


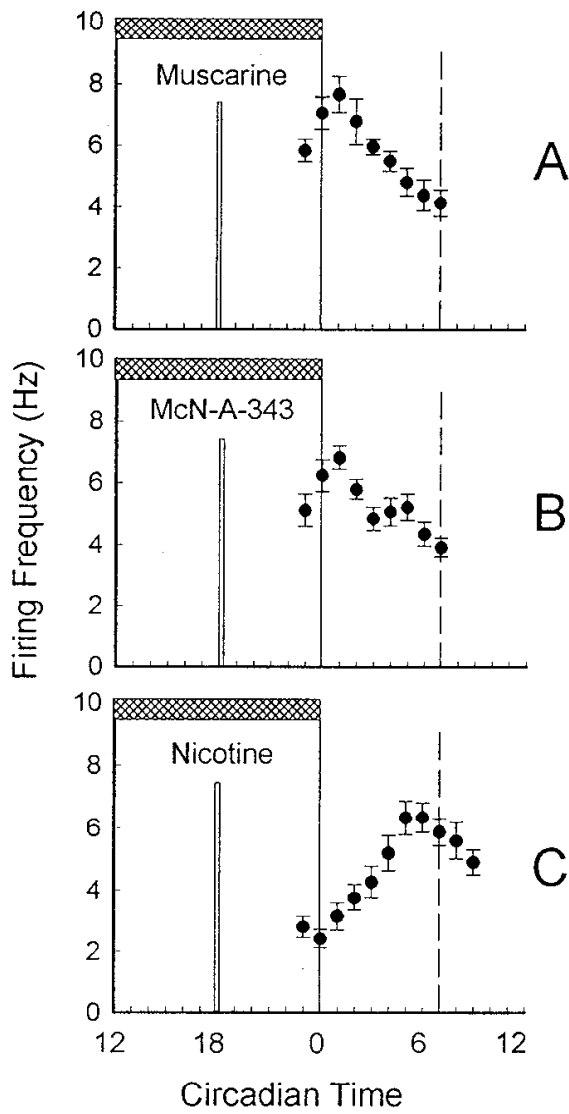

Figure 3. Effects of different cholinergic agonists applied at CT 18 on the time-of-peak in the SCN electrical activity rhythm. Results of three individual experiments are plotted as the running $2 \mathrm{hr}$ mean firing rates versus circadian time. Both $100 \mu \mathrm{M}$ muscarine $(A)$ and $10 \mu \mathrm{M}$ M1-selective agonist McN-A-343 $(B)$ induced phase advances of $\sim 6 \mathrm{hr}$ such that the peak on day 2 occurred at CT 1 . Nicotine $(100 \mu \mathrm{M} ; C)$ caused a $1 \mathrm{hr}$ phase advance. Symbols are as in Figure 1.

rine $\gg$ nicotine (Fig. 4). These experiments suggest that $\mathrm{mAChRs}$ mediate cholinergic regulation of the SCN rhythm at night.

\section{The effect of carbachol is not affected by nicotinic antagonists}

To confirm that an nAChR is not involved in the carbacholinduced phase shift, the effect of a competitive nicotinic antagonist, dihydro- $\beta$-erythroidine (DH $\beta E$ ), was examined. DH $\beta E$ (10 $\mu \mathrm{M})$ did not affect the time-of-peak of the SCN activity rhythm when bath-applied for $30 \mathrm{~min}$ surrounding CT 18 (peak of CT 6.8 $\pm 0.1 \mathrm{hr}, n=3$; Fig. $5 A$ ); nor did the $\mathrm{DH} \beta E$ treatment attenuate the $6 \mathrm{hr}$ phase advance induced by the $5 \mathrm{~min}$ carbachol $(100 \mu \mathrm{M})$ microdrop at the middle of the DH $\beta$ E pulse, at CT $18(+6.0 \pm 0.1$ hr, $n=3$; Fig. $5 B$ ). DH $\beta E$ has been shown to block nicotinic depolarizations of neurons in the brain slices at concentrations of 10 pM to $10 \mu \mathrm{M}$ (Wang et al., 1991; Stevens et al., 1993). Because $\mathrm{nAChRs}$ in central nervous system neurons are diverse, the effectiveness of a second nicotinic antagonist, $d$-tubocurarine, in blocking the effect of carbachol was tested. $d$-Tubocurarine may act at a different subtype of neuronal nAChR. Application of $d$-tubocurarine $(10 \mu \mathrm{M})$ for $30 \mathrm{~min}$ did not shift the SCN-activity rhythm (data not shown) or block the carbachol-induced phase advance at CT $18(n=3$; Fig. $5 C)$. These results demonstrate that

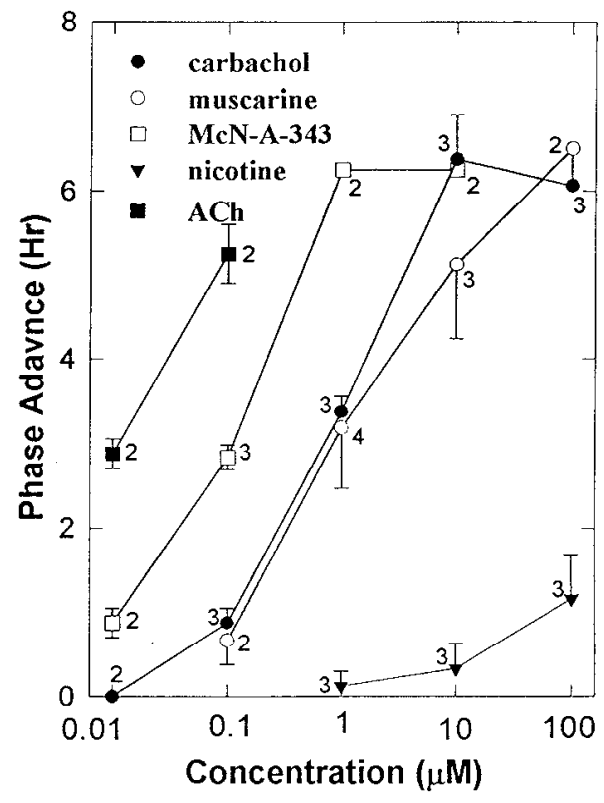

Figure 4. Dose-response curves for cholinergic agonists. Plotted are the averages \pm SEM of phase advances of the electrical activity rhythm induced at $\mathrm{CT} 18$ by 5 min exposure to various concentrations of each agonist. Treatments were applied as $1 \mu \mathrm{l}$ droplets so that the effective concentration likely was somewhat lower than that in the test solution. The number of experiments contributing to the mean phase shift produced at each concentration is labeled. ACh, the nonspecific agonist carbachol, and specific muscarinic agonists muscarine and M1-selective $\mathrm{McN}-\mathrm{A}-343$ induced dose-dependent phase shifts of the neuronal activity rhythm. Relative potency was as follows: $\mathrm{ACh}>\mathrm{McN}-\mathrm{A}-343>$ carbachol $\approx$ muscarine $\gg$ nicotine. The specific nicotinic agonist nicotine had no effect at concentrations of 1 and $10 \mu \mathrm{M}$, but induced a small phase advance $(+1.2 \pm 0.3 \mathrm{hr} ; n=3)$ at the highest treatment level $(100 \mu \mathrm{M})$.

the activation of $\mathrm{nAChR}$ is not required in the phase-shifting effect induced by carbachol.

\section{The effect of carbachol is blocked differentially by muscarinic antagonists}

To demonstrate further that carbachol acts via an mAChR to induce phase shifts and to explore the mAChR subtypes involved, we used three mAChR antagonists: atropine (nonselective), pirenzepine (M1-selective), and 4,2-(4,4'diacetoxydiphenylmethyl)pyridine (4-DAMP, M3-selective). When applied in the bath at the concentration of $0.1 \mu \mathrm{M}$ during the period surrounding carbachol treatment, atropine blocked the phase-advancing effect of carbachol $(100 \mu \mathrm{M})$ at Cl' $18(+1.1 \pm$ $0.3 \mathrm{hr}, n=3$; Figs. 6, 7). Atropine alone did not have an effect on the phase of the SCN rhythm at concentrations up to $1 \mu \mathrm{M}$. The M1-selective antagonist pirenzepine blocked the phase-advancing effect of carbachol $(100 \mu \mathrm{M})$ at CT 18 at a concentration of $1 \mu \mathrm{M}$ $(+0.67 \pm 0.08 \mathrm{hr}, n=3$; Figs. 6,7$)$, and less effectively at $0.1 \mu \mathrm{M}$ $(+3.9 \pm 0.1 \mathrm{hr} ; n=3)$. 4-DAMP, which is M3-selective, partially blocked the effect of carbachol at a concentration of $1 \mu \mathrm{M}(+4.1$ $\pm 0.1 \mathrm{hr}, n=3$; Fig. 7). The relative potency of the muscarinic antagonists in blocking the effect of carbachol is as follows: atropine $>$ pirenzepine $>4$-DAMP.

\section{DISCUSSION}

By evaluating the direct action of carbachol on the circadian rhythm of SCN neuronal activity, we have demonstrated that the $\mathrm{SCN}$ is itself sensitive to cholinergic phase resetting in a noctur- 

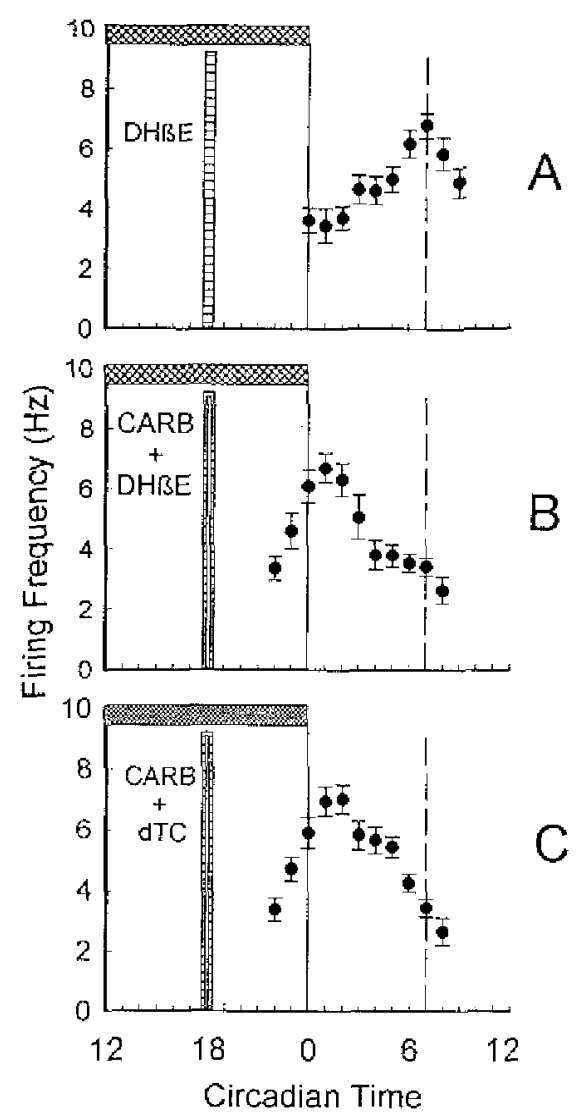

Figure 5. Fffects of the nicotinic antagonists $\mathrm{DH} \beta \mathrm{F}$ and $d$-tubocurarine. $A$, A 30 min bath application of DHBE $(10 \mu \mathrm{M})$ alone surrounding CT 18 had no effect on the activity thythm, $B$, The same DH $\beta E$ treatment did not prevent the $6 \mathrm{hr}$ phase advance induced by carbachol $(C A R B ; 100 \mu \mathrm{M}$ microdrop for 5 min at CT 18). C, Likewise, a 30 min bath application of $d$-tubocurarine (dTC) alone was without effect (data not shown), and it did not block the carbachol-induced $6 \mathrm{hr}$ phase advance (100 $\mu \mathrm{m}$ microdrop for $5 \mathrm{~min}$ at CT 18). Ventical bars with horizontal fills represent bath application of the antagonist; superimposed in $B$ and $C$ is the agonist treatment (vertical open bar). All other symbols are as in Higure 1.

nally restricted pattern and via an mAChR mechanism. The specific findings of our experiments were as follows. (1) The SCN circadian rhythm was phase-advanced up to $6 \mathrm{hr}$ by direct application of the cholinergic agonist carbachol to the $\mathrm{SCN}$ in the brain slice, and sensitivity to carbachol was circadian phase-dependent, occurring only during the subjective night. (2) This change in sensitivity is driven by endogenous clock mechanisms because it occurred in the isolatcd SCN in vitro. (3) The phase-shifting effect of carbachol was reproduced by $\mathrm{ACh}$ and two muscarinic agonists, muscarine and McN-A-343 (M1-selective), but not by nicotine. (4) The nAChR antagonist $\mathrm{DH} \beta \mathrm{E}(10 \mu \mathrm{M})$ did not affect the carbachol-induced phase advance. (5) Both the muscarinic antagonist atropine $(0.1 \mu \mathrm{M})$ and the $\mathrm{M} 1$-selective antagonist pirenzepine ( $1 \mu \mathrm{M})$ blocked the carbachol phase-shifting effect, whereas the M3-selective antagonist 4-DAMP $(1 \mu \mathrm{M})$ partially blocked the effect.

\section{Site of action}

The site at which carbachol affects circadian rhythms has resisted identification in wole-animal studies. The observation that intracerebroventricular injection of this cholinergic agonist causcs phase shifts has suggested that it acts within the SCN or at

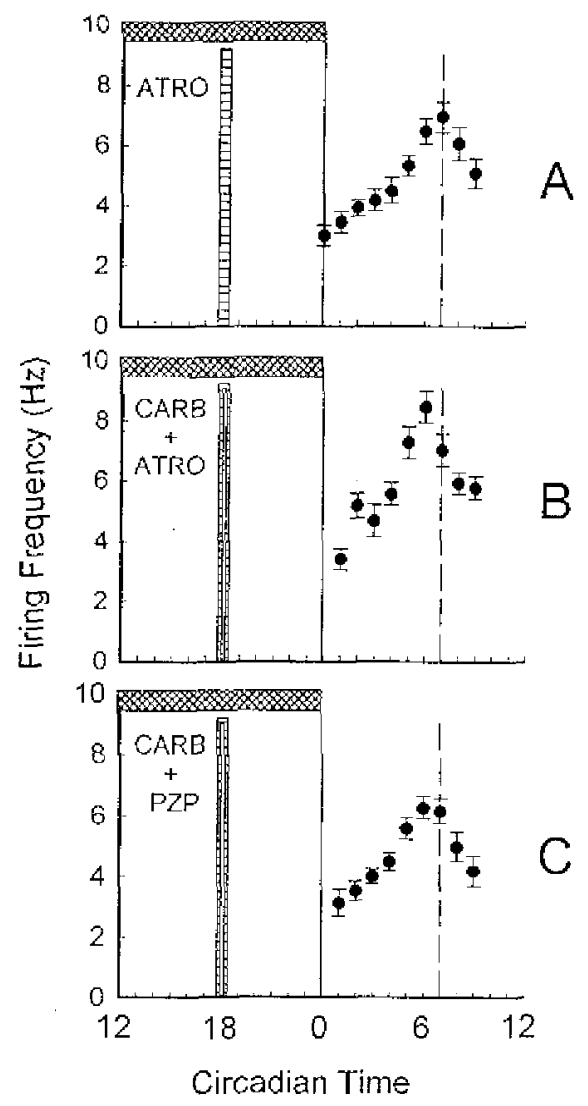

Figure 6. Blockade of carbachol-induced phase stift at $\mathrm{CT} 18$ by the muscarinic antagonists atropine and pirenzepine. $A, \mathrm{~A} 30$ min bath application of atropine $(A T R O ; 1 \mu \mathrm{M})$ alone surrounding CT 18 had no effect on the activity rhythm. $B$, Atropine $(0.1 \mu \mathrm{M})$ blocked the effect of a $5 \mathrm{~min}$ carbachol (CARB) microdrop (compare Fig. 1). $C$, The selective M1 antagonist pirenzepine $(P Z P ; 1 \mu \mathrm{M})$ also blocked the effect of carbachol at CT 18; pirenzepine alone had no effect on phase (data not shown). Symbols ate as in Figure 5 .

neurons that project to the SCN. In the present study, we demonstrated that carbachol can induce the same phase shift in the reduced slice preparation (limited to the SCN region) as it does in the hypothalamic slice. Therefore, carbachol must act directly on the SCN or in its immediate vicinity to induce the phase shift of the SCN neuronal activity thythm. Although we cannot exclude the possibility that carbachol acted on optic nerve terminals that remain in this reduced preparation, the most likely site of action is on SCN neurons, which express both muscarinic and nicotinic receptors (van der Zee et al., 1991).

Our study of the response of the SCN in vitro does not rule out the possibility that, in addition to acting directly on SCN neurons, carbachol injected intracerebroventricularly in vivo acts indirectly at different brain sites on neurons that project to the SCN to exert its effect on the behavioral circadian rhythms. Although early studies showed a light-like effect of intracerebroventricularly injected carbachol on wheel-running activity (Zatz and I Ierkenham, 1981), this was not repeated by others. Meijer et al. (1988) reported only phase advances, whereas Wee et al. (1992) observed much smaller amplitude of phase delays $(1.2 \mathrm{hr})$ induced by carbachol compared with phase advances $(4.3 \mathrm{hr})$. It is likely that the phase-delaying effect of carbachol seen in vivo is attributable to interaction with the retinohypothalamic tract (RHT) glutama- 


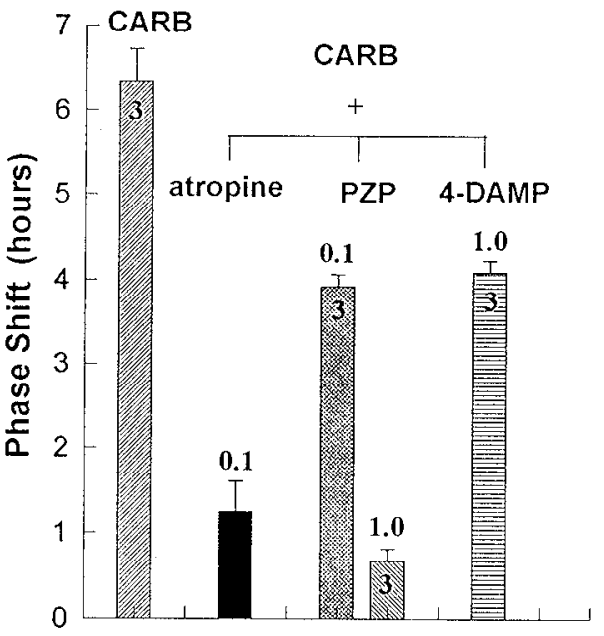

Figure 7. Relative potency of muscarinic antagonists in blocking the effect of carbachol at CT 18. Microdrop applications of $100 \mu \mathrm{M}$ carbachol $(C A R B)$ for $5 \mathrm{~min}$ induced a $6.3 \pm 0.2 \mathrm{hr}$ phase advance at C' $18(n=3)$. Muscarinic antagonists atropine $(0.1 \mu \mathrm{M})$, pirenzepine $(P Z P$, M1-selective; 0.1 and $1.0 \mu \mathrm{M})$, and 4-DAMP (M3-selective, $1.0 \mu \mathrm{M})$ were tested for their ability to block the carbachol-induced phase shift. Treatments and concentrations (in $\mu \mathrm{M}$ ) are labeled above each bar, and the number of replications $(n=3$ for each) is noted within each bar. Plotted are the means \pm SEM.

tergic system at sites not preserved well in the $\mathrm{SCN}$ brain slice (see below); this may modify its direct effect on the SCN pacemaker.

\section{Receptor subclasses and subtypes}

Carbachol is a nonselective cholinergic agonist that activates $m A C h R$ and $n A C h R$ subclasses. Previous studies of the effect of carbachol on the circadian rhythms in vivo were not conclusive as to receptor mechanism. The neurophysiological effects of cholinergic agents on SCN cells are also controversial. In the present study, we found that large-amplitude phase shifts induced by carbachol at CT 18 were produced by other cholinergics with the potency rank order as follows: $\mathrm{ACh}>\mathrm{McN}-\mathrm{A}-343>$ carbachol $\approx$ muscarine. These effects were not produced by nicotine (1-100 $\mu \mathrm{M})$. In addition, the muscarinic antagonists atropine and pirenzepine blocked the effect of $100 \mu \mathrm{M}$ carbachol at 0.1 and $1 \mu \mathrm{M}$ concentrations, whereas two nicotinic antagonists, $\mathrm{DH} \beta \mathrm{E}$ and $d$-tubocurarine, did not show any blocking effect at a concentration of $10 \mu \mathrm{M}$. 'These results demonstrate that $\mathrm{nAChR}$ activation is unlikely to contribute significantly to phase-shifting the circadian clock at CT 18; rather, mAChR-mcdiated mcchanisms can access the clock in its nocturnal state.

Our results differ from those of a recent study (Trachsel et al., 1995), which reported that the SCN rhythm was advanced by $2 \mathrm{hr}$ on average, independent of the CT, by nicotine. That paradigm examined the response to nicotine (10 $\mu \mathrm{m}$ for $1 \mathrm{hr}$ in the bath) applied across the circadian cycle. Our results do not dispute the report that nicoline can cause small phase advances. We also observed that nicotine (100 $\mu \mathrm{M}$ in a microdrop) caused a small phase advance (1.2 hr; see Fig. 4). However, they reported that carbachol was without effect. Although the discrepancy between the studies could be attributable to the differences in the methods of treatment and rat strains used, it should be noted that Trachsel et al. (1995) did not test the effect of carbachol between CT 15 and CT 21, which we demonstrate in this study to be the most sensitive period for carbachol to induce the phase shift.
Molecular cloning techniques have identified five genes $(\mathrm{m} 1$, $\mathrm{m} 2, \mathrm{~m} 3, \mathrm{~m} 4$, and $\mathrm{m} 5$ ) that encode distinct $\mathrm{mAChRs}$. The $\mathrm{m} 1$, $\mathrm{m} 2, \mathrm{~m} 3$, and $\mathrm{m} 4$ subtypes correspond to pharmacologically classified receptors $\mathrm{M} 1, \mathrm{M} 2, \mathrm{M} 3$, and $\mathrm{M} 4$, respectively (Watson and Abbot, 1992). Which of these might be expressed in the $\mathrm{SCN}$ is unknown. Our results suggest that carbachol activates an M1 mAChR in the SCN to phase-shift circadian rhythms. Although the mAChR subtypes have different sensitivity to the agonists and antagonists, they cannot be differentiated exclusively with these drugs. McN-A-343 is functionally potent in stimulating the M1 receptor but has less activity at M2 and M3; it shows little selectivity for these subtypes in the binding assay (Birdsall et al., 1983; Freedman et al., 1988; Lambrecht et al., 1993). In the present study, McN-A-343 mimicked the effect of carbachol at CT 18 at low concentrations and was more potent than carbachol and muscarine (see Fig. 4). Although it is possible that the phase-response curves for these cholinergic agonists are different so that their relative potencies could be different at circadian times other than CT 18 , the fact that the maximum phase shifts induced by these agonists at CT 18 are comparable does not support this possibility.

The M1-selective antagonist pirenzepine $(1 \mu \mathrm{M})$ blocked the phase-shifting effect of carbachol, whereas an M3 antagonist, 4-DAMP, partially blocked the effect at the same concentration. Although both pirenzepine and 4-DAMP have high affinities for the M1 subtype, with 4-DAMP slightly higher, pirenzepine has $>40$-fold lower affinities than does 4-DAMP for M2, M3, and M4 subtypes (Doods et al., 1987; Waelbroeck et al., 1990). Our results regarding potency rank order of the agonists and antagonists are mostly consistent with the interpretation that the phase-shifting effect of cholinergic agonists on the SCN activity rhythm is mediated via an M1 mAChR. However, it should be noted that these pharmacological data do not exclude the possibility of the involvement of an m5-like subtype of mAChR or a mixture of mAChR subtypes in regulating the SCN rhythm. Therefore, further studies are needed to identify with certainty the subtype(s) coupled with the $\mathrm{ACh}$ response when more selective agonists and antagonists become available.

\section{Phase-dependent sensitivity and relationship to light pulses in vivo}

Although the ACh agonist carbachol is the only agent that has been reported to "mimic" the phase-shifting effects of light when injected in vivo (Zatz and Brownstein, 1979; Zatz and Herkenham, 1981; Earnest and 'Iurek, 1983, 1985; Wee et al., 1992), the other evidence for the involvement of $\mathrm{ACh}$ in light-induced phase shifts is weak. This has becn referred to as "the carbachol paradox" by Colwell et al. (1993). Our results reveal that the SCN clock is sensitive to carbachol only during the subjective night of the circadian cycle. This timing of SCN sensitivity to carbachol is mostly consistent with results from in vivo experiments. However, the patterns of response in the two conditions differ. The response to intracerebroventricular injection of carbachol in vivo can be biphasic, with phase delays in wheel-running rhythms during early night and phase advances during late night. We did not observe a phase delay in vitro, which suggests that a phase delay induced by carbachol in vivo is not attributable to the direct effect of carbachol on the SCN. It has been shown that cholinergic fibers in the $\mathrm{SCN}$ region originate from cholinergic neurons in the cholinergic basal nuclear complex and in the mesopontine tegmentum. It has been speculated that these pathways provide feedback regulation of circadian timing relative to the state of sleep and wakefulness 
(Bina et al., 1993). Therefore, dircet stimulation of these areas in vivo would be predicted to cause phase shifts in behavioral circadian rhythms with patterns of sensitivity like the SCN sensitivity to carbachol.

Although cholinergic stimulation of the SCN in vitro does not generate the biphasic phase shifts induced by light, glutamate, the putative neurotransmitter in the RHT, does reproduce this effect. Glutamate applied in a similar paradigm to carbachol in the present study induced phase delays early and advances late in the subjective night in vitro (Ding et al., 1994). The pattern is very similar to the light pulse-induced changes in behavioral rhythm in rats (Summers et al., 1984). Because the sensitive periods of the SCN to phase-shifting by carbachol and glutamate are completely overlapping, and the effect of carbachol in vivo was blocked by an NMDA-receptor antagonist (Colwell et al., 1993), it is likely that an interaction exists between the cholinergic and glutamatergic systems within the SCN.

Such an interaction between ACh and glutamate may occur at the level of intracellular and/or intercellular signaling pathways involving nitric oxide (NO). NO donors mimic and NO synthase inhibitors block the effect of glutamate on SCN rhythms (Ding et al., 1994). This suggests the involvement of soluble guanylyl cyclase, which is activated by NO, and of the cGMP/cGMP. dependent protein kinase (PKG) system in glutamate-induced phase shifts. Interestingly, the phase-response curves for 8-bromo-cGMP, an analog of cGMP, and for carbachol overlap in time of sensitivity and are of the same amplitude (Prosser et al., 1990; Liu and Gillette, 1994). Furthermore, a specific inhibitor of PKG blocked the effect of 8-bromo-cGMP and the effect of carbachol (Liu and Gillette, 1994). This evidence points to the convergence of cholinergic and glutamatergic neurotransmissions at the CGMP-PKG signaling pathway in regulating the SCN response. However, glutamate must act via additional pathways: glutamate induces both delays and advances in the SCN circadian rhythm, whercas carbachol and cGMP analogs induce only advances, and these advances are significantly larger in amplitude than those of glutamate. Our results provide a basis for future study of the interaction between glutamatergic and cholincrgic systems at the SCN and may lead to a resolution of the carbachol paradox.

\section{REFERENCES}

Bina KG, Rusak B, Semba K (1993) Localization of cholinergic ncurons in the forebrain and brainstem that project to the suprachiasmatic nucleus of the hypothalamus in rats. J Comp Neurol 335:295-307.

Birdsall NJM, Burgen ASV, Hulme EC, Stockton JM (1983) The effect of McN-A-343 on muscarinic receptors in the cerebral cortex and heart. Br J Pharmacol 78:257-259.

Brownstein M, Kobayashi R, Palkovits M, Saavedra J (1975) Cholineacetyltransferase levels in diencephalic nuclei of the rat. J Neurochem $24: 35-38$.

Colwcll CS, Kaufman CM, Mcnaker M (1993) Phasc-shifting mechanisms in the mammalian circadian system: new light on the carbachol paradox. J Neurosci 13:1454-1459.

De Coursey PJ (1964) Function of a light response rhythm in hamsters. J Comp Physiol 63:189-196.

Ding JM, Chen D, Weber ET, Faiman LE, Rea MA, Gillette MU (1994) Resetting the SCN biological clock: mediation of nocturnal circactian shifts by glutamate and NO. Science 266:1713-1717.

Doods HN, Mathy MJ, Davidesko D, van Charldorp KJ, de Jonge A, van Zwieten PA (1987) Selectivity of muscarinic antagonists in radioligand and in vivo experiments for the putative $\mathrm{M} 1, \mathrm{M} 2$, and $\mathrm{M} 3$ receptors. $J$ Pharmacol Exp Ther 212:257-262.

Earnest DJ, Turek FW (1983) Rolc for acetylcholine in mediating cffects of light on reproduction. Science 219:77-79.
Earnest DJ, Turek FW (1985) Neurochemical basis for the photic control of circadian rhythms and seasonal reproductive cycles: role for acetylcholine. Proc Natl Acad Sci USA 82:4277-4281.

Freedman SB, Beer MS, Harley EA (1988) Muscarinic M1, M2 receptor binding: relationship with functional efficacy. Eur J Pharmacol 156:133-142.

Gillette MU (1986) The suprachiasmatic nuclei: circadian phase-shifts induced at the time of hypothalamic slice preparation are preserved in vitro. Brain Res 379:176 181.

Gillette MU, Prosser RA (1988) Circadian rhythm of the rat suprachiasmatic brain slice is rapidly reset by daytime application of cAMP analogs. Brain Res 474:348-352.

Gillette MU, Reppert SM (1987) The hypothalamic suprachiasmatic nuclei: circadian patterns of vasopressin secretion and ncuronal activity in vitro. Brain Res Bull 19:135-139.

Gillette MU, Medanic M, McArthur AJ, Liu C, Ding JM, Faiman LE, Weber ET, Tcheng TK, Gallman EA (1995) Intrinsic neuronal rhythms in the suprachiasmatic nuclei and their adjustment. In: Circadian clocks and their adjustment, pp 134-153. Chichester: Wilcy.

Green DJ, Gillette R (1982) Circadian rhythm of firing rate recorded from single cells in the rat suprachiasmatic slice. Brain Res 245:198-200.

Hatton GI, Doran AD, Salm AK, Tweedle CD (1980) Brain slice preparation: hypothalamus. Brain Res Bull 5:405-414.

Ichikawa T, Hirata Y (1986) Organization of choline acetyltransferasecontaining structures in the forcbrain of the rat. J Neurosci 6:281-292.

Kow L, Pfaff DW (1984) Suprachiasmatic neurons in tissue slices from ovariectomized rats: electrophysiological and neuropharmacological characterization and the effects of estrogen treatment. Rrain Res 297:275-286.

Lambrecht G, Moser U, Grimm U, Pfaff O, Hermanni U, Hilderbrandt C, Waclbrocck M, Christophe J, Mutschler E (1993) New functionally selective muscarinic agonists. Life Sci 52:481-488.

Liu C, Gillette MU (1994) Carbachol directly resets the circadian rhythm of $S \mathrm{CN}$ neuronal activity in vitro during subjective night through a cGMP/PKG-dependent mechanism. Soc Neurosci Abstr 20:159.

Meijer JH, van der Zee EA, Dietz M (1988) The effects of intraventricular carbachol injection on the free-running activity rhythm of the hamster. J Biol Rhythms 3:333-348.

Miller JD, Murakami DM, Fuller CA (1987) The response of suprachiasmatic neurons of the rat hypothalamus to photic and nicotinic stimuli. J Neurosci 7:978-986.

Nishino H, Koizumi K (1977) Responses of ncurons in the suprachiasmatic nuclei of the hypothalamus to putative transmitters. Brain Res 12():167-172.

Prosser RA, Gillette MU (1989) 'The mammalian circadian clock in the suprachiasmatic nuclei is reset in vitro by cAMP. J Neurosci 9:1073-1081.

Prosser RA, McArthur AJ, Gillctte MU (1990) cGMP induces phase shifts of a mammalian circadian pacemaker at night, in antiphase to cAMP effects. Proc Natl Acad Sci USA 86:6812-6815.

Rao ZR, Yamano M, Wanaka A, Tatehata T, Shiosaka S, Tohyama M (1987) Distribution of cholinergic neurons and fibers in the hypothalamus of the rat using choline acetyltransfurase as a marker. Neuroscicnce 20:923-934.

Rusak B, Bina KG (1990) Neurotransmitters in the mammalian circadian system. Annu Rev Neurosci 13:387-401.

Shibata S, Liou SY, Ueki S (1983) Different effects of amino acids, acetylcholine and monoamines on neuronal activity of suprachiasmatic nucleus in rat pups and adults. Neurosci Lett 39:187-192.

Stevens DR, Birnstiel S, Gerber U, McCarlcy RW, Greene RW (1993) Nicotinic depolarizations of rat pontine reticular formation neurons studied in vitro. Neuroscience 57:419-424.

Summers TL, Ferraro JS, McCormack CE (1984) Phase-response and Aschoff illuminance curves for locomotor activity rhythm of rat. Am J Physiol 246:R299.

Tago H, McGeer PL, Bruce G, Hersh LB (1987) Distribution of cholineacctyltransferase-containing neurons of the hypothalamus. Brain Res 415:49-62.

Trachsel L, Heller HC, Miller JD (1995) Nicotine phase-advances the circadian neuronal activity rhythm in rat suprachiasmatic nuclei explants. Neuroscience 65:797-8()3.

van den Pol AN, Tsujimoto KL (1985) Neurotransmitters of the hypothalamic suprachiasmatic nucleus: immunocytochemical analysis of 25 neuronal antigens. Neuroscience 15:1049-1086. 
van der Zee E^, Streefland C, Strosberg $\Lambda D$, Schroder H, Luiten PGM (1991) Colocalization of muscarinic and nicotinic receptors in cholinoceptive neurons of the suprachiasmatic region in young and aged rats. Brain Res 542:348-352.

Waelbroeck M, Tastenoy M, Camus J, Christophe J (1990) Binding of selective antagonists to four muscarinic receptors (M1 to M4) in rat torebrain. Mol Pharmacol 38:267-273.

Wang YT, Neuman RS, Bieger D (1991) Nicotinic cholinoceptor-mediated excitation in ambigual motoneurons of the rat. Neuroscience 40:759-767.

Watson S, Abbot A (1992) TIPS receptor nomenclature supplement. Trends Pharmacol Sci [Suppl] 12:19.

Wee BEF, Anderson KD, Kouchis NS, Turek FW (1992) Administration of carbachol into the lateral ventricle and suprachiasmatic nucleus (SCN) produces dose-dependent phase shifts in the circadian rhythm of locomotor activity. Neurosci Lett 137:211-215.

Zatz M, Brownstein MJ (1979) Intraventricular carbachol mimics the effects of light on the circadian rhythm in the rat pineal gland. Science 203:358-361.

Zatz M, Brownstein MJ (1981) Injection of $\alpha$-bungarotoxin near the suprachiasmatic nucleus blocks the effects of light on nocturnal pineal enzyme activity. Brain Res 213:438-442.

Zatz M, Herkenham MA (1981) Intraventricular carbachol mimics the phase-shifting effect of light on the circadian rhythm of wheel-running activity. Brain Res 212:234-238. 